ISSN 1991-8631

\title{
Variations micrométriques des tiges de jeunes plants de Acacia senegal (L.) Wildd dans différents régimes hydriques
}

\author{
Ibrahima KANE $^{1^{*}}$, Leonard Elie AKPO ${ }^{2}$, Michel GROUZIS ${ }^{3}$ and André BERGER ${ }^{4}$ \\ ${ }^{1}$ Université Cheikh Anta Diop-Dakar, Faculté des Sciences et Technologies de l'Education et de la Formation, \\ Département des Sciences de la Vie et de la Terre, Boulevard Habib Bourguiba, B.P. 5036 Dakar, Sénégal. \\ ${ }^{2}$ Universite Cheikh Anta Diop-Dakar, Faculté des Sciences et Techniques, Département de Biologie Végétale, \\ B.P. 5005 Dakar, Sénégal. \\ ${ }^{3}$ Mission IRD, Route d'Ambohipo, B.P. 434 Antananarivo, Madagascar. \\ ${ }^{4}$ CEFE/CNRS Laboratoire Ecophysiologie Montpellier. B.P. 5051, 34033 Montpellier, France. \\ *Auteur correspondant, E-mail : ibseydoukane@yahoo.fr
}

\section{RESUME}

L'influence des régimes d'alimentation hydrique sur les variations du diamètre des tiges et la réponse de jeunes plants de Acacia senegal (L.) Wildd après une réhydratation a été appréciée par la méthode des capteurs. 1-En condition d'alimentation hydrique optimale, Acacia senegal (L.) Wildd subit des variations nycthémérales: déshydratation au cours de la journée et double récupération hydrique pendant la nuit et en début de matinée. 2-L'état hydrique du sol détermine les variations d'amplitude des diamètres des jeunes plants qui sont d'autant plus importantes que la contrainte est sévère. 3-Les micro-variations des diamètres déterminent la croissance des jeunes plants qui est d'autant plus importante que le traitement est humide. 4-La réhydratation dans les traitements hydriques modéré et sévère se traduit par une reprise de la croissance avec des récupérations nocturnes en condition modérée.

(C) 2012 International Formulae Group. All rights reserved.

Mots clés : Acacia senegal, capteurs, traitements hydriques, variations de diamètre des tiges.

\section{INTRODUCTION}

L'utilisation des légumineuses pérennes dans la restauration des écosystèmes dégradés apparaît intéressante dans les zones où l'eau est un facteur limitant. La contrainte hydrique affecte en effet la survie des jeunes plants utilisés dans les programmes de reboisement. Ainsi, la réponse des jeunes plants de ces espèces aux conditions de déficit hydrique modéré ou permanent dépend du statut hydrique du sol et de la plante. Selon
Berger (1988), les variations journalières du stock d'eau peuvent provoquer des modifications physiologiques au sein $\mathrm{du}$ végétal qui sont fonction de l'amplitude et de la contrainte hydrique.

De nombreuses méthodes ont été utilisées pour déterminer l'effet de la contrainte hydrique sur la croissance et le fonctionnement hydrique des végétaux. Parmi celles-ci, les mesures de variation de diamètre ou micromorphométrie se sont répandues de 
plus en plus (Huguet, 1985 ; Katerji et al., 1990). En plus d'être un indicateur très commode (Schoch et al., 1987), elles constituent une voie prometteuse pour une perception globale des échanges hydriques au sein de la plante et présentent l'avantage d'être non destructifs (Huguet, 1985). Les variations de diamètre des tiges de jeunes plants, par rapport à la disponibilité de l'eau du sol, pourraient-elles donc constituer un critère permettant de préciser la croissance en vue de leur survie ultérieure dans des zones où la sécheresse est erratique ?

L'objectif de cette étude est de déterminer l'influence du régime d'alimentation hydrique sur les variations du diamètre et la réponse de jeunes plants de Acacia senegal L. après une réhydratation par la méthode des capteurs.

\section{MATERIEL ET METHODES} Matériel végétal

Le matériel végétal utilisé est constitué de jeunes plants de Acacia senegal (L.) Wildd issus de graines récoltées deux ans auparavant et provenant de Kidira (Est Sénégal). Elles ont été mises à notre disposition par le Centre National de Recherches Forestières (CNRF) de l'Institut Sénégalais de Recherches Agricoles (ISRA) et triées de façon à avoir un lot homogène.

\section{Dispositif expérimental}

L'essai a été réalisé dans 10 buses qui sont des tubes en PVC de $40 \mathrm{~cm}$ de haut et 40 $\mathrm{cm}$ de diamètre remplies d'un sol provenant de Dakar dont la texture est la suivante : $42 \%$ d'argile, $18 \%$ de limon et $27 \%$ de sable. Son Pf $2=3.29$ et le Pf $4.2=17.2$.

Les graines ont été trempées dans du $\mathrm{H}_{2} \mathrm{SO}_{4}$ pendant 15 min puis rincées plusieurs fois à l'eau stérile. A la suite de ce prétraitement, les graines ont été trempées dans de l'eau distillée pendant $24 \mathrm{~h}$. afin d'obtenir un gonflement.

Ainsi traitées, les graines sont mises à germer dans des boîtes de Pétri contenant de la gélose et placées à l'étuve à $30{ }^{\circ} \mathrm{C}$ pendant 12 à 16 h. Les boites de Pétri sont maintenues en position renversée afin que la radicule ne s'enfonce pas dans la gélose. Les graines ayant germé ont été transplantées et repiquées à raison de 2 par buses à $2 \mathrm{~cm}$ de profondeur. Un démariage a suivi 10 jours après. Les sols des buses ont été recouverts d'un film en polyéthylène noir et d' $1 \mathrm{~cm}$ de gravier afin de réduire au maximum les échanges hydriques entre surface du sol et atmosphère.

\section{Mesure du diamètre des tiges \\ Traitements appliqués}

Les régimes hydriques suivants ont été appliqués :

- un régime hydrique à capacité au champ

- un régime hydrique de contrainte modéré obtenu après arrêt de l'irrigation de 7 jours

- un régime hydrique de contrainte sévère obtenu après arrêt de l'irrigation de 15 jours.

Les teneurs en eau déterminées par la méthode gravimétrique étaient respectivement $29 \%$, $17 \%$ et $11 \%$. Pour chaque traitement, 3 pots ont été utilisés.

- une réhydratation par irrigation des régimes hydriques modéré et sévère à capacité au champ au dixième jour d'expérimentation à 12 heures.

\section{Méthodes de mesure}

Les mesures ont été effectuées sur des tiges de plants âgés de 3 mois par des capteurs de flux de déplacements linéaires à induction différentielle munis de portecapteurs (Huguet, 1985). Les signaux des capteurs ont été enregistrés en continu toutes les 30 minutes dans une centrale d'acquisition de données Micrologger 21X. Les enregistrements sont transférés dans un microordinateur et traités au laboratoire. 
Le capteur a été placé à $10 \mathrm{~cm}$ du sol toujours avant la première ramification. Les mesures du diamètre de la tige sont réalisées sur des plants qui ont un diamètre compris entre 5 et $10 \mathrm{~mm}$ et une hauteur de 50 à 80 $\mathrm{cm}$.

\section{Traitement des données}

Le traitement des données s'est fait comme suit :

La variation réelle enregistrée du diamètre de la tige $=$ variation mesurée par le capteur + dilatation thermique de l'ensemble support capteur + dilatation thermique de l'organe. La dilatation thermique de l'ensemble support capteur a été corrigée à partir des mesures de variations de températures enregistrées par les thermocouples et par un coefficient de dilatation de l'ensemble capteur pour des températures variables. $\mathrm{La}$ dilatation thermique de l'organe a été négligée. Les valeurs des diamètres sont transformées en $\mu \mathrm{m}$ à partir de l'étalonnage et mises à 0 au début de l'analyse.

Les contractions maximales journalières sont déterminées à partir de la différence entre le diamètre maximal et le diamètre minimal.

\section{RESULTATS}

Variations du diamètre des tiges des plants en conditions optimales pendant trois jours

Les variations de diamètre des tiges des plants en conditions optimales, suivies pendant 3 journées successives, sont portées dans la Figure 1.

Pour chaque journée de mesure, une contraction des tiges qui s'accompagne d'une diminution de leur diamètre s'amorce à $11 \mathrm{~h}$. Cette contraction atteint une valeur minimale à $17 \mathrm{H} 30 \mathrm{~min}$, à $15 \mathrm{~h} 30 \mathrm{~min}$ et à $17 \mathrm{~h}$ respectivement aux premier, deuxième et troisième jours.
A cette phase de diminution qui correspond à une contraction, fait immédiatement suite une phase de dilatation correspondant à une phase de récupération jusqu'au lendemain. Cette phase de récupération qui dure $20 \mathrm{~h}$ comprend une étape de récupération au cours de la nuit jusqu'à 4 h, suivie d'une deuxième plus rapide qui se poursuit même au moment de l'ensoleillement. L'amplitude entre le diamètre maximal atteint en début de journée et le diamètre minimal pendant la journée représente la contraction maximale journalière. Elle est respectivement de 136 $\mu \mathrm{m}, 135 \mu \mathrm{m}$ et $154 \mu \mathrm{m}$. La différence entre les diamètres maximaux de 2 journées consécutives qui traduit la croissance de la plante montre une augmentation du diamètre de $89 \mu \mathrm{m}$ et de $54 \mu \mathrm{m}$.

\section{Variations du diamètre des tiges dans les 3 traitements}

Deux journées de mesures ont été choisies pour caractériser les variations de diamètre des tiges dans les 3 traitements hydriques (Figure 2). En conditions optimales, les phases de dilatation et de contraction décrites précédemment sont observées.

Dans le traitement hydrique modéré en fin de journée et en début de matinée, des phases de récupération sont constatées alors qu'en période d'ensoleillement des séries de contraction et de dilatation sont observées. Pendant la période nocturne une seule phase de récupération est notée.

Dans le traitement hydrique sévère, aucune récupération n'est observée pendant la nuit et au cours de la journée, les phases de contraction et de dilatation sont beaucoup plus marquées et plus fréquentes qu'en contrainte modérée.

Les moyennes des contractions maximales journalières sont plus élevées en conditions optimales que dans les traitements 
soumis à la contrainte. Elles sont cependant plus accentuées en contrainte hydrique sévère qu'en contrainte modérée (Tableau 1)

Suivi de la croissance à partir de la variation du diamètre des tiges dans les 3 traitements hydriques

Dans le traitement hydrique optimal, on observe des phases de dilatation et de contraction avec une augmentation d'amplitude permettant d'atteindre un niveau supérieur à la veille sauf entre les $6^{\text {ème }}$ et $7^{\text {ème }}$ jours (Figure 3). Cette croissance se traduit par la valeur élevée $(a=1.25)$ de la pente de la droite d'ajustement.

Dans le traitement modéré, est observée une augmentation de l'amplitude pendant les 6 premiers jours traduisant une légère croissance. Lorsque le déficit hydrique s'accentue il ya diminution de l'amplitude. Cette croissance est très faible comme le montre la pente de la droite d'ajustement $(\mathrm{a}=0.20)$

Dans le traitement de contrainte hydrique, une diminution de l'amplitude s'observe progressivement à partir du quatrième jour. La pente négative $(\mathrm{a}=-0.13)$ dans le traitement de contrainte hydrique sévère montre que la croissance ne s'effectue pas.

\section{Variation du diamètre des tiges après une réhydratation}

L'irrigation se traduit par une récupération qui débute à 12 h 30 min et qui se maintient jusqu'au jour suivant à $9 \mathrm{~h}$ dans le traitement hydrique modéré (Figure 4).

Dans le traitement hydrique sévère nous observons une forte récupération entre $13 \mathrm{~h} 30 \mathrm{~min}$ et $14 \mathrm{~h} 30 \mathrm{~min}$. Les phases de récupération pendant les périodes ensoleillées semblent être suffisantes pour assurer la croissance des jeunes plants. Aucune récupération nocturne n'a été enregistrée dans ce traitement.

Dans le traitement hydrique modéré, la récupération pendant la nuit est importante et, au cours des périodes d'insolation, des phases de contraction et de dilatation beaucoup moins fréquentes sont observées. Ces récupérations rapides de la plante dans les 2 traitements entraînent une reprise de la croissance matérialisée par les pentes de 1.6 et de 2.8 respectivement en condition de contrainte et en condition modérée.

Tableau 1 : Moyennes des contractions maximales journalières dans les trois régimes hydriques chez de jeunes plants de Acacia senegal (L.) Wildd.

\begin{tabular}{cccc}
\hline & Traitement optimal & $\begin{array}{c}\text { Contrainte } \\
\text { modérée }\end{array}$ & Contrainte sévère \\
\hline $\begin{array}{c}\text { Moyennes contractions } \\
\text { maximales }(\mu \mathrm{m})\end{array}$ & $156 \mathrm{a}$ & $73 \mathrm{c}$ & $109 \mathrm{~b}$ \\
\hline
\end{tabular}

Les différences sont significatives au seuil de 5\% (test de Newman-Keuls). 


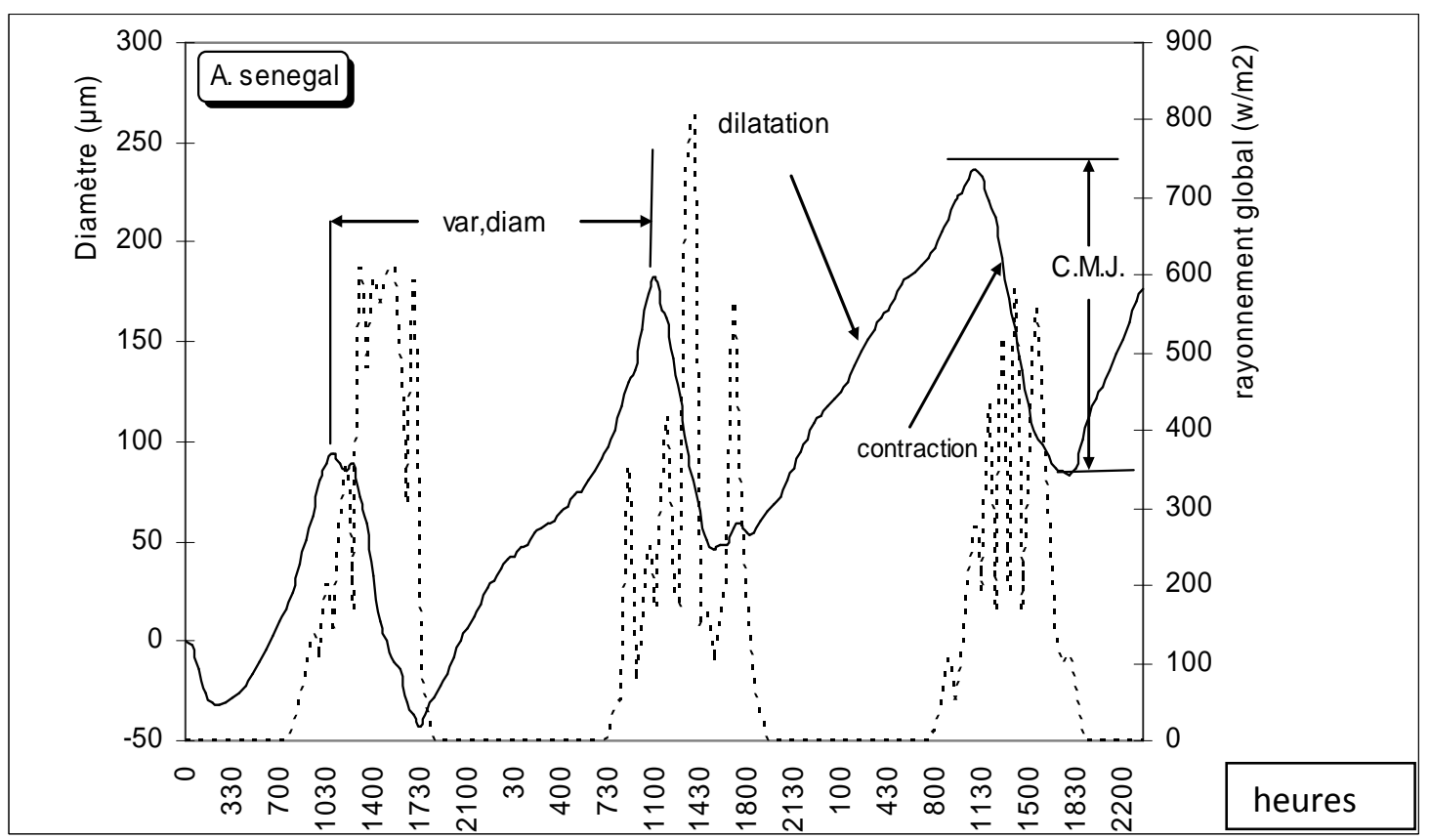

Figure 1: Variations du diamètre de la tige en conditions optimales d'alimentation hydrique au cours de 3 jours successifs à différentes heures.

C.M.J. = Contraction maximale journalière

Var.diam $=$ Variation du diamètre

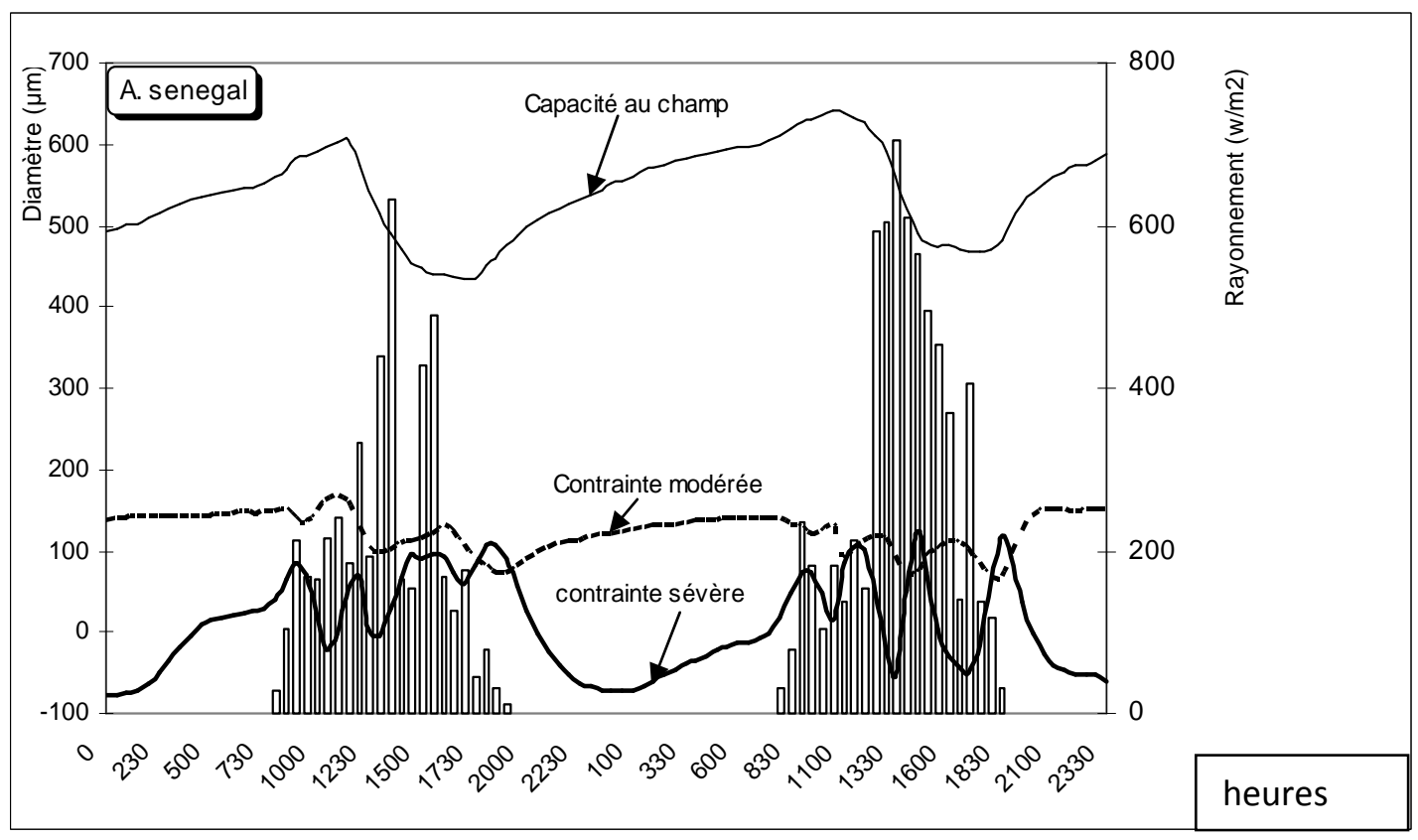

Figure 2 : Comparaison des variations du diamètre des tiges de jeunes plants de Acacia senegal (L.) (Wildd) dans les trois traitements hydriques au cours de 2 jours successifs à différentes heures. 


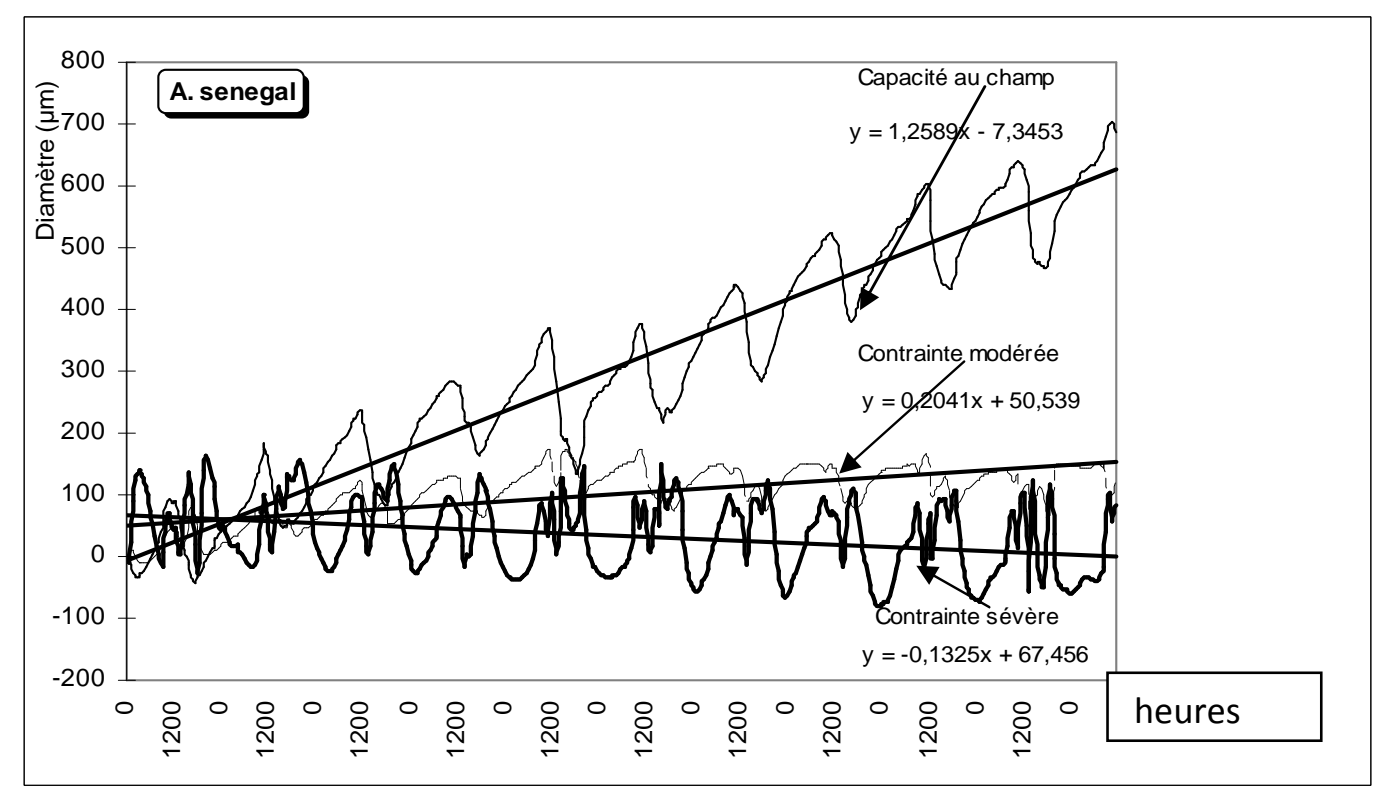

Figure 3 : Variations du diamètre des tiges et phénomène de croissance chez de jeunes plants d'Acacia senegal (L.) Wildd. dans les trois régimes hydriques.

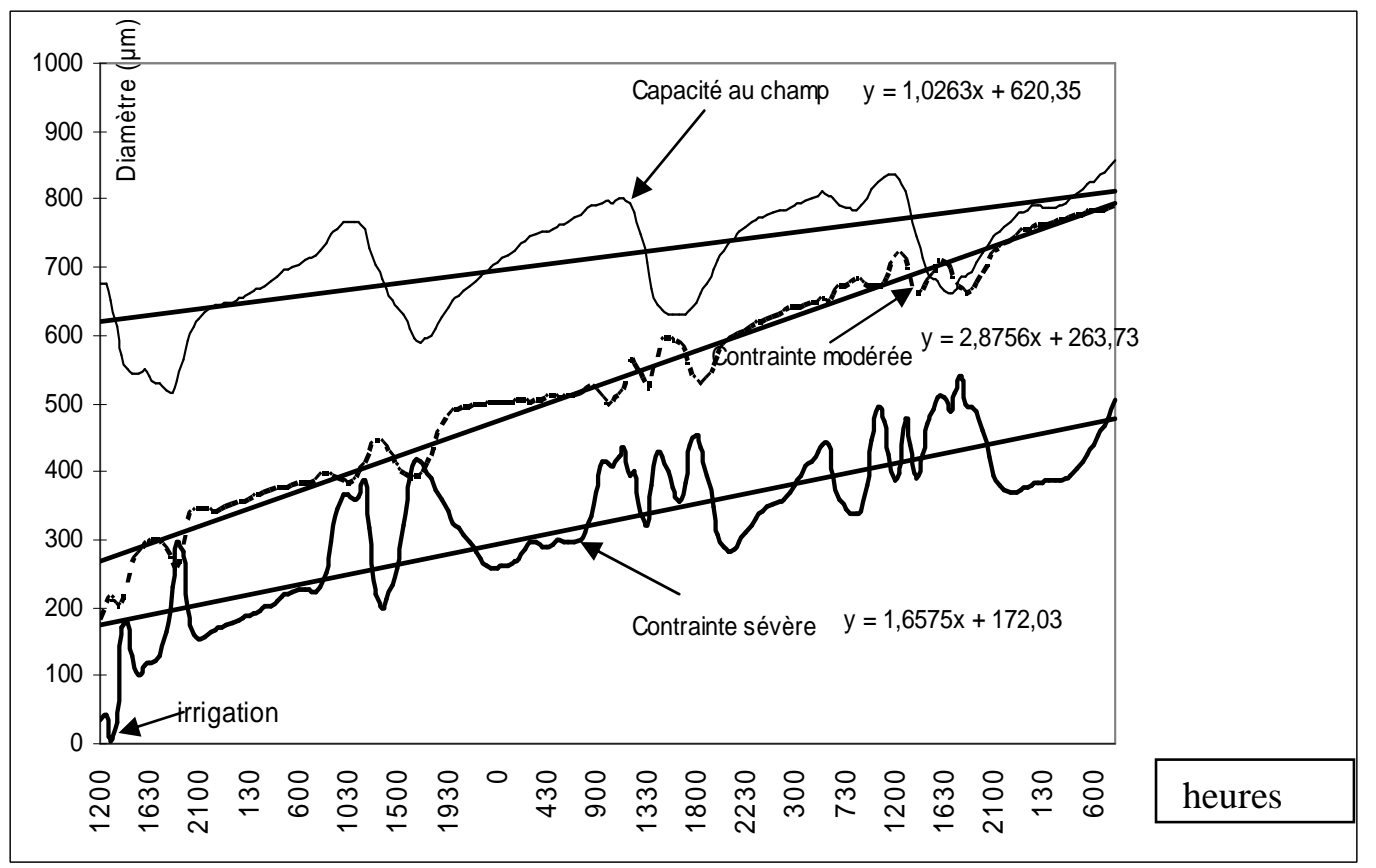

Figure 4 : Evolution du diamètre des tiges de Acacia senegal L. après irrigation des traitements hydriques modéré et sévère à différentes heures. 


\section{DISCUSSION}

Les variations micrométriques des tiges des jeunes plants de Acacia senegal en conditions d'alimentation hydrique optimale montrent des variations nycthémérales qui se manifestent par une phase de contraction qui traduit une transpiration et qui se déroule exclusivement pendant les périodes ensoleillées et une phase de réhydratation à deux temps :

- un premier temps s'effectuant en fin de journée et au cours de la nuit, qui consisterait à une reconstitution des réserves ;

- un deuxième temps se poursuivant même pendant les périodes d'insolation, qui représenterait le transfert des produits de photosynthétats dans les compartiments de la plante.

Pour Jarvis (1975), ces variations du diamètre de la tige sont essentiellement dues à une variation d'épaisseur de la zone cambium + liber. Molz et Klepper (1972) ont d'ailleurs montré que les contractions et les dilatations venaient essentiellement des variations de la teneur en eau des tissus corticaux, c'est-à-dire des tissus élastiques externes au xylème formé du cambium, du phloème et des cellules immatures du proto-xylème et que le xylème ne subissait pas de variations de diamètre. Huguet (1985) précise également que les contractions portent essentiellement sur la partie extra-cambiale (phloème, tissus corticaux etc.), le xylème étant totalement rigide.

Ces séquences événementielles aujourd'hui reconnues comme classiques ont été mises en évidence par de nombreux auteurs (Huguet, 1985; Garnier, 1985 ; Schoch et al., 1987; Selles, 1988 ; Berger, 1988; Katerji et al., 1990). Au cours des périodes ensoleillées, s'observe un flux d'eau de l'écorce vers le xylème dû à la réduction du potentiel xylémien par rapport à celui du phloème entraînant une contraction de la tige. En fin de journée, durant la nuit et en début de matinée, la situation inverse se produit. Le potentiel hydrique du phloème est plus bas par rapport à celui du xylème; l'eau transite du xylème vers le phloème. Cette phase correspond à une récupération hydrique durant laquelle la plante reconstitue ses réserves.

Les micro-variations des diamètres des tiges déterminent donc une croissance qui traduit une augmentation de taille ou du nombre de cellules grâce à l'arrivée d'eau et des assimilas (Huguet, 1985). Ce même auteur souligne que cette expansion ne peut s'exprimer que lorsque le potentiel hydrique n'est pas trop bas en fin d'après-midi, la nuit ou en début de matinée. Ces phases de dilatation et de contraction s'accompagnent d'une reprise permettant d'atteindre un niveau supérieur à la veille. Cette augmentation du diamètre de la tige que nous avons constatée est également présentée comme un critère définissant la croissance générale de la plante (Schoch et al., 1987). L'évolution nette du diamètre maximal journalier qui est la différence entre les diamètres maximaux mesurés avant le début de la contraction sur 2 journées consécutives varie suivant les journées de $89 \mu \mathrm{m}$ à $54 \mu \mathrm{m}$. Ces variabilités ont été également mises en évidence chez le Pêcher (Garnier, 1985 ; Selles, 1988). Urban et al. (1994) signalent également chez de jeunes fraisiers une augmentation de 40 à 45 $\mu \mathrm{m}$.

Nos résultats montrent que lorsque les jeunes plants de Acacia senegal sont placés en conditions hydriques modérée et sévère, les phases de contraction et de dilatation observées pendant la journée sont d'autant plus accentuées et fréquentes que la contrainte hydrique est sévère. Les moyennes de contractions maximales journalières passent de $73 \mu \mathrm{m}$ à $109 \mu \mathrm{m}$ avec des différences significatives. Cette accentuation de la contraction a été mise en évidence chez des plantes d'aubergine par Katerji et al. (1990) et ce phénomène s'accentuait du fait du déficit hydrique imposé à la plante. Au cours des périodes ensoleillées, la plante transpire puis a tendance à fermer ses stomates pour réduire les pertes en eau car la disponibilité en eau du sol est limitée. Pendant la fermeture, la plante se réhydrate et lorsque l'état hydrique est 
reconstitué, elle transpire à nouveau. Selon Huguet (1985), ces récupérations partielles indiquent que les plants se trouvent soumis à une contrainte hydrique dans les traitements à conditions hydriques modérée et sévère. Simmoneau et al. (1993) ainsi que Urban et al. (1994) montrent d'ailleurs que contraction et dilatation sont liées au contenu hydrique de la plante. Amiglio (1991) précise qu'en situation de sécheresse édaphique, les réserves en eau du tronc participent à la transpiration de la plante. Huguet (1985) a montré en effet des résultats similaires sur le citronnier. Cependant, pour ce dernier, les réserves hydriques sont apportées par les fruits et les organes ligneux. Ceci serait dû à un effet hydraulique de réhydratation partielle des tissus pendant la nuit.

Lorsque la contrainte hydrique est modérée, une légère croissance est observée comme l'indique la pente de la droite d'ajustement. Hsiao et al. (1976) précisent d'ailleurs qu'en cas de déficit modéré, les processus de croissance sont touchés en premier. Lorsque la contrainte hydrique est sévère, une diminution de la croissance est constatée comme le montre la pente négative de la droite d'ajustement. Ce résultat confirme ceux de Selles (1988), Berger et Selles (1990) et Kozlowski (1991). La diminution du diamètre des tiges est également nette. Cette réduction absolue du diamètre des troncs est un phénomène courant observé chez les arbres en période de forte sécheresse (Aussenac et al., 1984). En condition hydrique limitant, elle est une conséquence d'une déshydratation progressive des tissus en réponse au déficit hydrique imposé à la plante.

Il semble ainsi exister une réelle réduction de la vitesse de croissance et de la variation du diamètre des tiges des jeunes plants de Acacia senegal en rapport avec le déficit hydrique du sol.

Après irrigation, une reprise de la croissance et des périodes de récupération sont observées chez les plants placés dans les deux traitements hydriques. Ces phénomènes ont pu être montrés par Reich et Borchert
(1984) sur des arbres tropicaux et sur le Noyer (Amiglio, 1991). La récupération se fait quelques heures après l'irrigation et correspond à une réhydratation des tissus élastiques et à une nouvelle croissance cellulaire (Lassoie, 1979; Aussenac et al., 1984). Elle semble être une constante puisque après un déficit hydrique du sol. Schoch et al. (1987) et Selles (1988) observent à la suite d'une pluie ou d'une irrigation chez les espèces forestières des récupérations très rapides. Celles-ci se manifestent chez les jeunes plants de Acacia senegal (L.) Wildd. leur permettant ainsi de pouvoir supporter les déficits hydriques dans les zones arides. Cependant, elles ne se traduisent pas automatiquement par un retour au cycle normal probablement à cause de la résistance variable à l'entrée de l'eau dans la plante.

Dans cette étude, la relation entre les variations micrométriques des diamètres de tiges de jeunes plants de Acacia senegal L. et la disponibilité de l'eau du sol a permis de préciser les variations nycthémérales: une déshydratation et une double réhydratation. De plus, lors d'une irrigation des sols en contraintes hydriques modéré ou sévère, une reprise de la croissance est observée. Ces processus traduisent une croissance générale des jeunes plants indispensable à la réussite de leur implantation en conditions naturelles. L'étude pourrait être combinée à des indicateurs de l'état hydrique de la plante pour pouvoir déterminer s'il existe des valeurs minimales de variations de diamètres susceptibles de limiter la croissance du jeune plant.

\section{REFERENCES}

Amiglio T. 1991. Relations hydriques chez le jeune noyer de l'échelle de la journée à celle de l'année en liaison avec quelques aspects de la physiologie de l'arbre. Thèse Université Blaise Pascal (Clermont II), p. 99.

Aussenac G, Granier E, Ibrahim M. 1984. Influence du dessèchement du sol sur le fonctionnement hydrique et la croissance 
$\mathrm{du}$ Douglas (Pseudotsuga menziesii (Mirb) Franco). Oecol. Plant, 5: 241-253.

Berger A. 1988. La circulation de l'eau à travers la plante. La Houille Blanche, 3(4): 227-233.

Berger A and Selles G. 1990. Diurnal fruit shrinkage: a model. In Water Transport in Plants Under Climatic Stress, Borghetti M, Grace J, Raschi A (Eds). Vallombrosa: Firenze, Italy; 261-268.

Garnier E. 1985. Dessèchement du sol et indicateurs physiologiques de l'état hydrique chez le pêcher : application au déclenchement de l'irrigation. Thèse Docteur - ingénieur, Université Montpellier, p. 82.

Huguet JG. 1985. Appréciation de l'état hydrique d'une plante à partir des variations micrométriques de la dimension des fruits ou des tiges au cours de la journée. Agronomie, 5(8): 733-741.

Hsiao TC, Fereres E, Acevedo E, Henderson DW. 1976. Water stress and dynamics of growth and yield of crop plants. In Water and Plant Life. Problems and Modern Approaches, Lange OL, Kappen L, Shulze ED (eds). Springer Verlag: New York; 281-305.

Jarvis PG. 1975. Water Transfert in Plants. Heat and Mass Transfer in the Biosphere I. Transfer Processes in Plant Environment, de Vries DA, Afgan NH (eds). John Wiley Press: New York; 369394.

Katerji N, Schoch PG, Rimgoto P, L'Hotel JC. 1990. Diagnostic des périodes de contrainte hydrique chez des plantes d'aubergine cultivées en serre, au moyen des microvariations des tiges. Agronomie, 10: 541-549.
Kozlowski TT, Kramer PJ, Pallardy S. 1991. The Physiological Ecology of Woody Plants. Academic Press: New York.

Lassoie JP. 1979. Stem dimensional fluctuations in Douglas-fir of different crown classes. Forest Science, 25: 132144.

Molz FJ, Klepper B. 1972. Radial propagation of water potential in stems. Agronomy Journal, 64: 469-473.

Reich PB, Borchert R. 1984. Water stress and tree phenology in a tropical dry forest in the lowlands of Costa Rica. Journal of Ecology, 72: 61-74

Schoch PG, Katerji N, Rimgoto P, Tchamitchian M, Malet P, L'Hotel JC, Dauney MC.1987. Influence du niveau d'alimentation hydrique sur les variations du diamètre des tiges, du potentiel hydrique, de la résistance stomatique, de la transpiration et de la photosynthèse de l'aubergine (Solanum melangene L.). Agricultural and Forest Meteorology, 40: 89-104.

Selles G. 1988. Effet de la contrainte hydrique sur la variation journalière du diamètre des troncs et des fruits. Application au déclenchement de l'irrigation du pêcher. Thèse ENSA, Montpellier, p.162.

Simmoneau T, Habib R, Goutouly JP and Huguet JG. 1993. Diurnal changes in stem diameter depend upon variation in water content: direct evidence in Peach trees. Journal of Experimental Botany, 44(260): 615-621.

Urban L, Fabret C, Barthelemy L. 1994. Interpreting changes in stem diameter in rose plants. Physiologia Plantarum, 92: 668-674. 